\title{
Plankton communities in oxbow lakes of the River Vistula (Oświęcim Basin) with bottom sediments heterogeneously contaminated with heavy metals
}

\author{
Joanna Gadzinowska \\ Institute of Nature Conservation, Polish Academy of Sciences, \\ Mickiewicza 33, 31-120 Krakow, Poland, e-mail: gadzinowska@iop.krakow.pl
}

\begin{abstract}
This study presents plankton communities (phytoplankton and zooplankton) in the three reservoirs of the River Vistula's oxbow lake near Zator (Oświęcim Basin). Selected physicochemical parameters of the oxbow lake waters and the bottom sediments were analysed. Aquatic ecosystems in the investigated area have been heterogeneously contaminated with heavy metals (mostly by zinc, cadmium, lead and chromium), because of contaminated waters of the Vistula, and with manganese and iron. The highest differences were observed between Krajskie and Miejsce oxbow lakes among chlorides and the conductivity of the water and among Cr and Fe in the bottom sediments. This indicates a strong influence of the River Vistula. The plankton structure was different in each of the oxbow lakes. The index of taxa similarity showed also differences in the structure of plankton in oxbow lakes, which was convergent with the chemical parameters of oxbow lakes. The index of taxa similarity was similar for all three oxbow lakes only for one group of algae (Chlorophyta) and one group of plankton animals (Rotatoria). Lower similarity was shown between one oxbow lake impacted by the River Vistula (Krajskie) and another oxbow lake completely separated from the River Vistula (Oko).
\end{abstract}

Key words: oxbow lake, heavy metals, bottom sediments, plankton, similarity index

\section{Introduction}

Planktonic organisms, having short life cycles and high intensity of multiplying, quickly respond to changes in the aquatic environment (Cottenie et al. 2001). The composition of plankton varies depending on the changes in the water habitats, and differs even in reservoirs with similar environmental conditions. Close adjacent reservoirs may show diverse structures of microorganism communities. This differentiation is often caused by various factors, e.g. fluctuations of water level, location of the water in relation to the impact of winds, movement of water, depth of reservoir, presence or absence of thermal stratification in the reservoir, cover of macrophytes, fish density, and physico-chemical factors not only of water but also of sediments (Cottenie et al. 2001). Studies examining the effects of bottom sediments varying in quality on planktonic assemblages are fairly poor, but a few of them have demonstrated the impact of heavy metals on these aquatic organisms (Skowroński et al. 2002; Atici et al. 2008; Atici et al. 2010; Wilk-Woźniak et al. 2011).

Heavy metals may affect many metabolic processes in live organisms. The adverse effect is mainly based on the inhibition of processes such as cellular respiration and photosynthesis. In the case of plant organisms it can cause the degradation of the photosynthetic pigments, interfere with cellular transport processes and the ionic balance in the cells (Skowroński et al. 2002). These negative processes can lead to stunted growth and to algal dying (Skowroński and Rzeczycka 1980).

However, there are species that are abundant in the water habitats with bottom sediments contaminated with heavy metals. It is a proven fact that metal contamination of bottom sediments did not affect the abundance of phyto- and zooplankton, but changed their structure and composition (Wilk-Woźniak et al. 2011). These habitats were populated by species 
resistant to toxic metals and elimination of sensitive species were observed. The species that are characterized by greater tolerance to toxic metals are species belonging to diatoms, green algae, or cyanobacteria (Skowroński et al. 2002). However, recent studies showed the toxic effects of heavy metals on complex communities of phytoplankton through measurements of oxidative stress biomarkers (Vega-Lopez et al. 2013). Although phytoplankton suffers oxidative stress elicited by metals, it is possible to counter this damage only through antioxidant defences (Vega-Lopez et al. 2013). Reactions of particular species may be different because of the degree and type of contamination of the habitat and the local conditions of physico-chemical properties $(\mathrm{pH}$, water temperature, available light and nutrient content: Skowroński et al. 2002; Pawlik-Skowrońska 2002a). The impact of heavy metals on zooplankton relies on inducing a teratogenic effect, which is manifested as deformed spines, blades and carapaces of planktonic animals (WilkWoźniak et al. 2011).

There are many areas in Poland, especially in the southern part, contaminated with heavy metals, because of mining and processing operations of nonferrous ores (e.g. $\mathrm{Zn}, \mathrm{Pb}, \mathrm{Cu}$ ) and in highly urbanized areas. Examples of contaminated water habitats are rivers in the valley of the Upper River Vistula, polluted by coal mines and also by the steel and chemical industries. Pollution is observed in the form of elevated loads of suspended particulate matter, sulphates, chlorides and nitrates, and high concentrations of heavy metals in sediments (Ciszewski and Czajka 2009).

The aim of this study was to estimate the composition of plankton communities in the River Vistula's oxbow lakes near Zator (Oświęcim Basin), in relation to sediments heterogeneously contaminated with heavy metals by the waters of the River Vistula.

\section{Study area}

The investigations were conducted on the oxbow lakes "Wiślisko" located in the area near Zator of the municipality Spytkowice (District Wadowice, Lesser Poland Voivodeship). The oxbow lakes comprise a complex of three reservoirs: a) Miejsce $\left(50^{\circ} 0^{\prime} 55.63^{\prime \prime} \mathrm{N}\right.$, $\left.\left.19^{\circ} 30^{\prime} 48.09^{\prime \prime} \mathrm{E}\right), \mathrm{b}\right)$ Oko (500'30.21” N,19³1'15.62” E) and c) Krajskie ( $\left.50^{\circ} 0^{\prime} 48.69^{\prime \prime} \mathrm{N}, 1^{\circ} 31^{\prime} 46.76^{\prime \prime} \mathrm{E}\right)$. These oxbow lakes are impacted by the waters of the River Vistula during periods of flooding, but Krajskie is also particularly endangered by the impact of the Vistula due to a persistent connection to a small river channel. The area of the oxbow lakes is as follows: Miejsce - 17 ha, Oko - 7 ha, Krajskie - 28 ha. All of them are shallow, up to $2.5 \mathrm{~m}$ in the area of Miejsce near the concave edge of the bend, undercut in the past by the River Vistula (Zając et al. 2013).

\section{Materials and methods}

The samples of bottom sediments were taken in July 2011 (29 samples) (Fig. 1). For chemical analysis the upper layer of sediments with a thickness of about $10 \mathrm{~cm}$ was taken. Material from the surface layer of the bottom was collected using a Van Veen type probe. In the Environmental Analysis Laboratory of the Faculty of Geology, Geophysics and Environmental Protection at the University of Science and Technology in Krakow the grain fraction $<63 \mu \mathrm{m}$ was separated by wet sieving. Then the content of heavy metals $(\mathrm{Cd}$, $\mathrm{Cr}, \mathrm{Cu}, \mathrm{Pb}, \mathrm{Zn}, \mathrm{Fe}$ and $\mathrm{Mn}$ ) was examined using the AAS flame method (spectrometer ICE 3400/3500 of Unicam Ltd) (Gadzinowska 2012).

Samples for analysis of the physico-chemical parameters of water were taken in April, June and September 2011 (Fig. 1). In each of these three seasons, 4 samples were taken from Miejsce (altogether 12 samples), 1 sample from Oko (altogether 3 samples) and 3 samples from Krajskie (altogether 9 samples). The single sampling point in Oko was chosen due to its small size, low morphological diversification and expected small variations in the physicochemical parameters. Water parameters were measured in situ by a Multiparameter Water Quality Sonde YSI 6600 V2: pH $(\mathrm{pH})$, conductivity (PEW), the content of chloride, nitrate, ammonium, and oxygen saturation. Samples for phosphate analysis were taken from the upper layer (1m) of each of the oxbow lakes, immediately transported to the laboratory, and analysed according to Hermanowicz et al. (1999).

Samples for phyto- and zooplankton qualitative analysis were taken in April, June and September 2011, each time samples from two points in Miejsce, one point in Oko, and one point in Krajskie (from the upper layer of the centre point of the reservoirs, $1 \mathrm{~m}$ ). Samples for phytoplankton analysis were taken by a 5 litre bathometer and concentrated by a plankton net $\# 10 \mu \mathrm{m}$ and for zooplankton by a plankton net \#50 $\mu \mathrm{m}$. Fresh samples were analysed under a Zeiss-Jenaval light microscope. The remains of samples of phytoplankton were fixed with Lugol's iodine and samples 


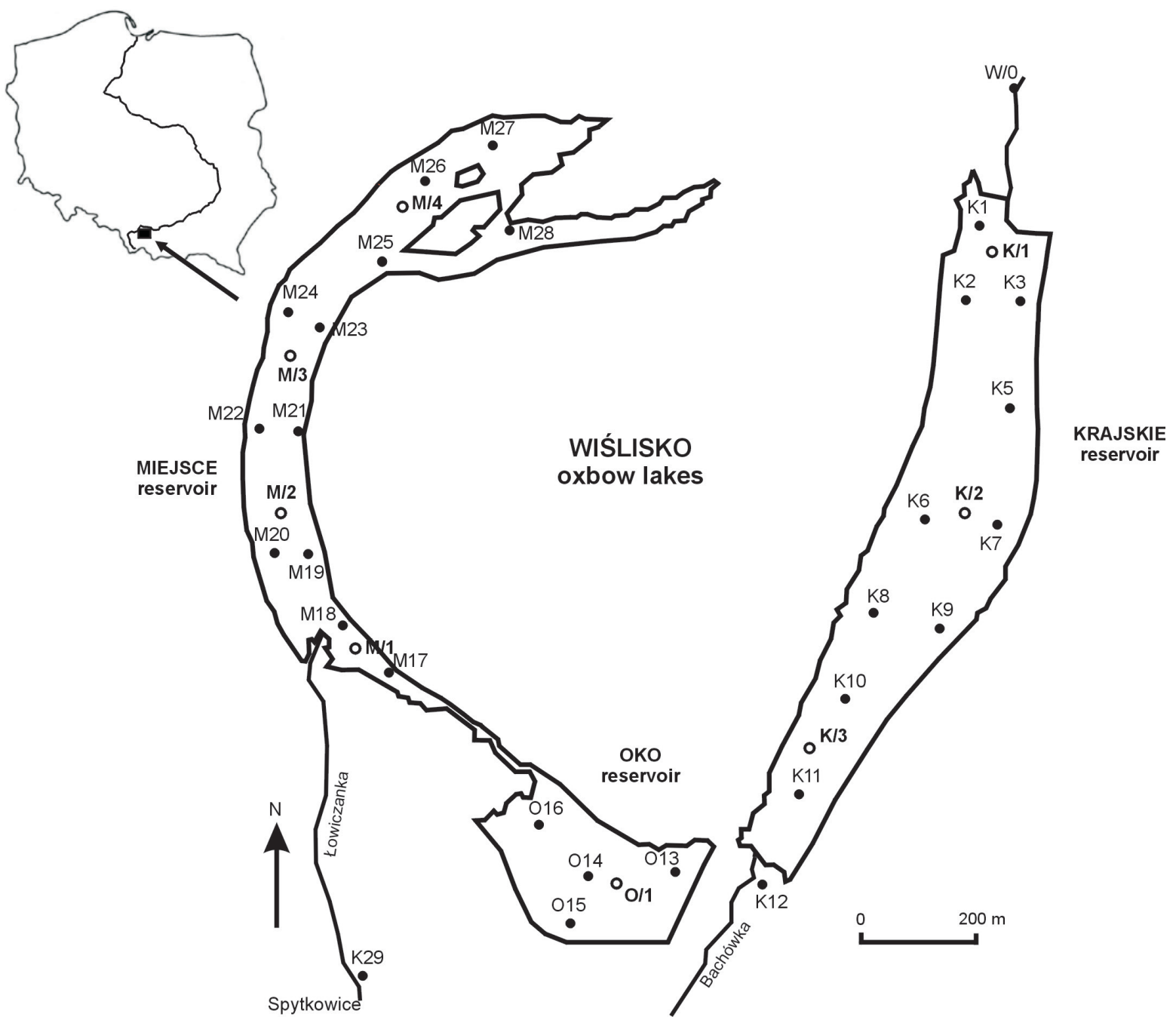

Fig. 1. Localization of sampling points of waters and bottom sediments in the "Wiślisko" oxbow lakes: $\mathrm{K}_{\mathrm{i}}$ - samples taken from Krajskie oxbow lake (excluding $\mathrm{K} 29$ ); $\mathrm{O}_{\mathrm{i}}$ - samples taken from Oko oxbow lake; $\mathrm{M}_{\mathrm{i}}$ - samples taken from Miejsce oxbow lake. Description: K1K12; O13-O16; M17-M28 - sediment sampling points; K29 - sediment sampling point in the Łowiczanka stream; W/0 - sediment sampling point in the River Vistula; $\mathrm{K} / 1, \mathrm{~K} / 2, \mathrm{~K} / 3, \mathrm{O} / 1, \mathrm{M} / 1, \mathrm{M} / 2, \mathrm{M} / 3, \mathrm{M} / 4$ - sampling points for analyses of physico-chemical parameters of waters; $\mathrm{K} / 2, \mathrm{O} / 1, \mathrm{M} / 2$ and $\mathrm{M} / 3$ - sampling points for phyto- and zooplankton analyses

of zooplankton were fixed by $4 \%$ formalin to preserve for further, more detailed qualitative analysis. For the keys used for the taxonomical analyses of phytoplankton, see e.g. Wilk-Woźniak (2009) and of zooplankton Pociecha et al. (2010). For all three reservoirs the taxonomic similarity factor was calculated, based on the most diverse groups: Cyanobacteria, Bacillariophyceae, Euglenophyta, and Chlorophyceae (phytoplankton) and Rotifera and Cladocera, and Copepoda (zooplankton). Other groups were represented only by one species or no representative was observed. The model for the taxonomic similarity factor is as follows: $\mathrm{P}=2 \mathrm{z} /(\mathrm{x}+\mathrm{y})$, where: $\mathrm{P}-$ taxonomic similarity factor; $\mathrm{x}$ - number of species in first reservoir; $y$ - number of species in second reservoir; $\mathrm{z}$ - number of common species in both reservoirs (Greig-Smith 1983).

To find the significant differences between water parameters and the concentration of metals in the bottom sediments of three oxbow lakes, the KruskalWallis test with post-hoc Dunn's test was performed using Statistica 8.0 software.

\section{Results and discussion}

Bottom sediments of the studied oxbow lakes, compared with the limit values for the background water sediments in Poland (Lis and Pasieczna 1995), showed high contamination with heavy metals (main- 
ly zinc, cadmium and lead) (Table 1). The highest contamination of these metals was found in samples taken in the area of Krajskie, which is directly connected by the channel with the River Vistula and in the sample from the River Vistula. The highest concentration of manganese and iron was found in Oko and Miejsce, oxbow lakes which have no direct connection with the River Vistula. A similar observation of a very high concentration of both metals was noted in the Vistula oxbow lake in Niepołomice forest, cut from the river ca. 200 years ago (Wilk-Woźniak et al. 2013). It seems that a high concentration of $\mathrm{Mn}$ and $\mathrm{Fe}$ in the bottom sediments of oxbow lakes is not a result of the impact of the river, but of these metals being natural elements of sediments.

Contaminated sediments can be harmful for biological supplies of surface waters. Notably dangerous for living organisms are heavy metals with high mobility, especially Cd, Zn, Cu and Mn (Helios-Rybicka 1986, 1991). Unfortunately, in Poland, no standards for the assessment of harmful concentrations of metals in sediments for living organisms have been developed yet. The only method, the value of PEL (Probable Effect Level) (MacDonald et al. 2000)) is used for the purpose of monitoring the quality of surface waters (conducted by the Polish Geological Institute). Adverse effects evolved by contaminated sediments to aquatic organisms are often observed (GIOŚ 2011). The threshold values of PEL [ $\mathrm{mg} \mathrm{kg}^{-1}$ ] are as follows: $\mathrm{Zn}-315 ; \mathrm{Pb}-91.3$; $\mathrm{Cu}-197 ; \mathrm{Cd}-3.53$ (Smith et al. 1996).

The concentration of zinc, lead and copper in the studied oxbow lakes did not show values higher than the threshold; therefore, these metals may not be harmful for aquatic organisms. Only the concentration of cadmium from Krajskie reservoir showed a higher value of PEL. In water ecosystems, cadmium is pres- ent in exchangeable mineralogical forms and has the highest toxicity effect among all of the analysed metals (Bajkowska 2012). This suggests that, in "Wiśliska" oxbow lakes, only cadmium may exert harmful effects on water organisms, especially those living in or on sediments like benthic organisms and mussels. For planktonic organisms heavy metals can be dangerous when they are remobilized from the sediments into water as a result of chemical or biochemical processes. However, recent studies show that mixtures of heavy metals, including micronutrients such as $\mathrm{Zn}, \mathrm{Fe}, \mathrm{Cu}$, Mn could be more toxic than Cd (Vega-Lopez et al. 2013; Skowroński et al., 2002). The highest impact on the mobility of heavy metals is caused by a change in $\mathrm{pH}$. In the conditions of low $\mathrm{pH}$ of the water, the metals may be released from clay minerals and hydroxides of Fe. Also changes in the oxidation-reduction potential lead to changes in the binding forms of metals in the solid phase and a further drop in $\mathrm{pH}$ increases their mobility (Łojan 2008). However, the high values of $\mathrm{pH}$ of the waters of all of the oxbow lakes (Table 2) indicate that the problem of increasing the mobilization of heavy metals does not exist as yet. The $\mathrm{pH}$ did not vary among the seasons and among the oxbow lakes, but other chemical factors varied depending on season and reservoir.

Oxygen saturation of the water in all three oxbow lakes showed differences mostly in the summer, in both vertical and horizontal planes. During the summer the water saturation of surface layers ranged from 77.4 to $123.0 \% \mathrm{O}_{2}$ (Krajskie o.l.); $58.0 \% \mathrm{O}_{2}$ (Oko o.l.); and from 62.3 to $97.4 \% \mathrm{O}_{2}$ (Miejsce o.l.). However, at the depth less than $1 \mathrm{~m}$ above the bottom, deficiency of oxygen was observed (Zając et al. 2013). In such specific conditions, release of metal ions from the bottom sediments to the water is possible. Therefore, the main

Table 1. The content of heavy metals in samples of bottom sediments in the investigated oxbow lakes (Gadzinowska 2012). Description: $\mathrm{K}$ - samples taken from the Krajskie oxbow lake; $\mathrm{O}$ - samples taken from the Oko oxbow lake; $\mathrm{M}$ - samples taken from the Miejsce oxbow lake; K29 - sample taken from the Łowiczanka Stream; W/0 - sample taken from the Vistula River

\begin{tabular}{|c|c|c|c|c|c|c|c|c|c|c|c|c|c|c|}
\hline \multirow{3}{*}{$\begin{array}{l}\text { Samples } \\
\text { of bottom } \\
\text { sediments }\end{array}$} & \multicolumn{2}{|c|}{$\mathrm{Cd}$} & \multicolumn{2}{|c|}{$\mathrm{Cr}$} & \multicolumn{2}{|c|}{$\mathrm{Cu}$} & \multicolumn{2}{|c|}{$\mathrm{Pb}$} & \multicolumn{2}{|l|}{$\mathrm{Zn}$} & \multicolumn{2}{|l|}{$\mathrm{Mn}$} & \multicolumn{2}{|c|}{$\mathrm{Fe}$} \\
\hline & $\min -\max$ & average & $\min -\max$ & average & $\min -\max$ & average & $\min -\max$ & average & $\min -\max$ & average & $\min -\max$ & average & $\min -\max$ & average \\
\hline & \multicolumn{2}{|c|}{ [mg kg-1] } & \multicolumn{2}{|c|}{$\left[\mathrm{mg} \mathrm{kg}^{-1}\right]$} & \multicolumn{2}{|c|}{ [mg kg-1] } & \multicolumn{2}{|c|}{$\left[\mathrm{mg} \mathrm{kg}^{-1}\right]$} & \multicolumn{2}{|c|}{$\left[\mathrm{mg} \mathrm{kg}^{-1}\right]$} & \multicolumn{2}{|c|}{$\left[\mathrm{mg} \mathrm{kg}^{-1}\right]$} & \multicolumn{2}{|c|}{ [\% weight] } \\
\hline K1-K12 & $1.03-8.10$ & 4.7 & $21.8-42.5$ & 34 & $22.2-46.7$ & 35.9 & $38.1-139.9$ & 92.3 & 242.3-851.4 & 548.1 & $275.8-729$ & 542.3 & $1.91-4.36$ & 3.52 \\
\hline 013-016 & $2.10-3.14$ & 2.7 & $25.1-45$ & 35.7 & $22.3-37.2$ & 27.6 & $48.6-87.8$ & 62.6 & 279.1-344 & 306.2 & 679.3-10163 & 3104 & $3.87-4.54$ & 4.20 \\
\hline M17-M28 & $0.58-7.21$ & 3.2 & $19.1-55$ & 42.5 & $15.2-44.2$ & 29.8 & $28.8-155.5$ & 88.2 & $183.1-407.1$ & 309.1 & $376.2-15643$ & 2757.2 & $2.09-6.41$ & 4.65 \\
\hline K29 & 1.68 & 1.68 & 38.3 & 38.3 & 35.9 & 35.9 & 95.1 & 95.1 & 682.2 & 682.2 & 593.8 & 593.8 & 4.01 & 4.01 \\
\hline W/O & 6.28 & 6.28 & 42.5 & 42.5 & 40.8 & 40.8 & 108.5 & 108.5 & 823.8 & 823.8 & 729 & 729 & 4.36 & 4.36 \\
\hline
\end{tabular}


Table 2. Physico-chemical parameters of waters of the investigated oxbow lakes: Krajskie, Oko and Miejsce (by Ciszewski et al. 2012, after modification). Description of the sampling points: K/1-K/3 - Krajskie oxbow lake; O/1- Oko oxbow lake; M/1-M/4 - Miejsce oxbow lake

\begin{tabular}{|c|c|c|c|c|c|c|c|c|c|c|c|c|c|c|c|c|c|c|c|c|c|c|c|c|}
\hline \multirow{3}{*}{ Parameter } & \multicolumn{8}{|c|}{ APRIL } & \multicolumn{8}{|c|}{ JUNE } & \multicolumn{8}{|c|}{ SEPTEMBER } \\
\hline & \multicolumn{3}{|c|}{ KRAJSKIE } & \multicolumn{2}{|l|}{ OKO } & \multicolumn{3}{|c|}{ MIEJSCE } & \multicolumn{3}{|c|}{ KRAJSKIE } & \multicolumn{2}{|l|}{ OKO } & \multicolumn{3}{|c|}{ MIEJSCE } & \multicolumn{3}{|c|}{ KRAJSKIE } & \multicolumn{2}{|l|}{ OKO } & \multicolumn{3}{|c|}{ MIEJSCE } \\
\hline & $\mathrm{K} / 1$ & $\mathrm{~K} / 2$ & $\mathrm{~K} / 3$ & O/1 & $M / 1$ & $\mathrm{M} / 2$ & $M / 3$ & $\mathrm{M} / 4$ & $\mathrm{~K} / 1$ & $\mathrm{~K} / 2$ & $\mathrm{~K} / 3$ & O/1 & $\mathrm{M} / 1$ & $\mathrm{M} / 2$ & $M / 3$ & $\mathrm{M} / 4$ & $\mathrm{~K} / 1$ & $\mathrm{~K} / 2$ & $\mathrm{~K} / 3$ & $0 / 1$ & M/1 & $\mathrm{M} / 2$ & $M / 3$ & $M / 4$ \\
\hline $\mathrm{pH}$ & 7.45 & 7.46 & 7.42 & 7.29 & 7.49 & 7.75 & 7.79 & 7.29 & 7.74 & 7.54 & 7.48 & 7.41 & 7.49 & 7.58 & 7.43 & 7.5 & 7.44 & 8.2 & 7.8 & 7.42 & 7.73 & 7.81 & 7.72 & 7.78 \\
\hline $\begin{array}{l}\text { Conductivity } \\
{\left[\mu \mathrm{S} \mathrm{cm}^{-1}\right]}\end{array}$ & 463 & 503 & 411 & 329 & 339 & 307 & 310 & 329 & 1211 & 939 & 505 & 343 & 339 & 338 & 339 & 343 & 367 & 501 & 539 & 311 & 309 & 309 & 307 & 306 \\
\hline $\begin{array}{l}\text { Chlorides } \\
{\left[\mathrm{mg} \mathrm{dm}^{-3}\right]}\end{array}$ & 47.65 & 58.12 & 32.64 & 15.14 & 13.25 & 13.58 & 15.85 & 15.13 & 145.86 & 102.24 & 59.75 & 13.53 & 13.25 & 13.81 & 13.72 & 13.76 & 29.045 & 73.93 & 83.28 & 15.24 & 15.00 & 14.47 & 12.85 & 15.51 \\
\hline $\begin{array}{l}\text { Nitrates } \\
{\left[\mathrm{mg} \mathrm{dm}^{-3}\right]}\end{array}$ & 2.345 & 2.161 & 2.554 & 0.378 & 0.632 & 0.518 & 0.343 & 0.375 & 1.132 & 0.992 & 0.662 & 0.793 & 0.632 & 0.702 & 0.858 & 0.786 & 0.157 & 0.054 & 0.06 & 0.239 & 0.57 & 0.444 & 0.1 & 0.165 \\
\hline $\begin{array}{l}\text { Phosphates } \\
{\left[\mathrm{mg} \mathrm{dm}^{-3}\right]}\end{array}$ & 0.002 & 0.011 & 0.001 & 0.001 & 0.000 & 0.000 & 0.001 & 0.001 & 0.008 & 0.006 & 0.003 & 0.006 & 0.000 & 0.002 & 0.001 & 0.001 & 0.046 & 0.019 & 0.038 & 0.017 & 0.026 & 0.037 & 0.019 & 0.017 \\
\hline $\begin{array}{l}\text { Ammonium } \\
{\left[\mathrm{mg} \mathrm{dm}^{-3}\right]}\end{array}$ & 0.085 & 0.091 & 0.22 & 0.01 & 0.38 & 0.005 & 0.008 & 0.01 & 0.164 & 0.109 & 0.193 & 0.037 & 0.38 & 0.32 & 0.342 & 0.29 & 0.091 & 0.001 & 0.009 & 0.058 & 0.002 & 0.006 & 0.030 & 0.006 \\
\hline
\end{tabular}

negative effect of heavy metals on microorganisms should be visible during the summer. In spring and autumn the regular oxygen saturation was observed in all reservoirs $\left(100 \% \mathrm{O}_{2}\right.$ Krajskie and Oko, and $60 \%$ $\mathrm{O}_{2}$ Miejsce). Lack of deficiency of oxygen during the spring and autumn allows the absence of migration of metal ions into the water to be stated. Thus, the probability of adverse effects induced by heavy metals on aquatic organisms is low.

Conductivity and chlorides showed relatively high values in Krajskie, but in Miejsce and Oko both factors showed rather low values, as characteristic for waters of good quality status. The highest value of conductivity was found in June at point $\mathrm{K} / 1$, close to the channel connecting the oxbow lake with the River Vistula $\left(1211 \mu \mathrm{S} \mathrm{cm}^{-1}\right.$ ) (Zając et al. 2013).

Krajskie oxbow lake also showed the highest concentration of nitrates and ammonia in the water during spring and summer, but during autumn, the highest nitrates concentrations were found for Miejsce oxbow lake, and ammonia concentrations for Oko oxbow lake. Similar values of phosphates were found for all three oxbow lakes. Although many studies showed that an increase in nutrient levels influences the phytoplankton composition (Watson et al. 1997), it seems that similar levels of phosphates were not the most important fact impacting the plankton composition. A more important factor appeared to be the concentration of chloride in Krajskie. It is known that salinity influences diatom physiology directly by exerting an osmotic stress, which may also affect spe- cies composition indirectly via interaction with other factors (Shobert 1974).

The Kruskal-Wallis test with post-hoc Dunn's test showed significant differences between Oko and Miejsce oxbow lakes in $\mathrm{pH}$ (Kruskal-Wallis $\mathrm{p}=0.0396$, post-hoc Dunn z=0.0344), Krajskie and Miejsce oxbow lakes in conductivity (Kruskal-Wallis $\mathrm{p}=0.0003$, posthoc Dunn $z=0.00018$ ), Krajskie and Miejsce oxbow lakes in chlorides (Kruskal-Wallis $\mathrm{p}=0.0003$, post-hoc Dunn $\mathrm{z}=0.00021$ ), Krajskie and Miejsce oxbow lakes in $\mathrm{Cr}$ concentration in bottom sediments (KruskalWallis $\mathrm{p}=0.0534$, post-hoc Dunn $\mathrm{z}=0.0491$ ) and Krajskie and Miejsce oxbow lakes in Fe concentration in bottom sediments (Kruskal-Wallis $\mathrm{p}=0.0468$, post-hoc Dunn $\mathrm{z}=0.0427$ ).

Among the phytoplankton of the studied oxbow lakes different algal groups were observed: Cyanophyceae, Bacillariophyceae, Dinophyceae, Euglenophyta, Chrysophyceae and Chlorophyceae. The total number of phytoplankton species found in 2011 was as follows: Krajskie - 65 species; Oko - 66; Miejsce - 88. Phytoplankton species composition was characteristic for each of the reservoirs (Table 3). In Krajskie, a large variety of Euglenophyta species was found, represented mainly by species of the genus Euglena and Trachelomonas. Oko reservoir, which is the least contaminated part of the oxbow lakes, was characterized by the dominance of Bacillariophyceae species. In previous years three species were found, classified in the "Red list of algae in Poland" as endangered (Siemińska et al. 2006): Cymbella cf. proxima Reimer, 
Table 3. Phytoplankton species noted in oxbow lakes "Wiśliska" in 2011

\begin{tabular}{|c|c|c|c|}
\hline Group, species & Krajskie & Oko & Miejsce \\
\hline \multicolumn{4}{|l|}{ Cyanoprokaryota } \\
\hline Anabaena danica_(Nygaard) J.Komárková-Legnerová \& P.Eloranta & & & $\mathrm{X}$ \\
\hline Aphanizomenon gracile (Lemmermann) Lemmermann & & & $\mathrm{X}$ \\
\hline Aphanizomenon sp. & & & $\mathrm{X}$ \\
\hline Aphanocapsa sp. & & $\mathrm{X}$ & $\mathrm{X}$ \\
\hline Chroococus limneticus Lemmermann & $\mathrm{X}$ & $\mathrm{X}$ & $\mathrm{X}$ \\
\hline Leptolyngbya sp. & $\mathrm{X}$ & & $\mathrm{X}$ \\
\hline Microcystis aeruginosa Kützing & & & $\mathrm{X}$ \\
\hline Microcystis wesenbergii (Komárek) Komárek & & & $\mathrm{X}$ \\
\hline Oscillatoria limosa C.Agardh ex Gomont & & & $\mathrm{X}$ \\
\hline Planktothrix agardhii (Gomont) Anagnostidis \& Komárek & $\mathrm{X}$ & & $\mathrm{X}$ \\
\hline Raphidiopsis cf. mediterranea Skuja & $\mathrm{X}$ & & \\
\hline Snowella sp. & $\mathrm{X}$ & & $\mathrm{X}$ \\
\hline Trichodesmium sp. & $\mathrm{X}$ & & \\
\hline Woronichinia naegeliana (Unger) Elenkin & & & $\mathrm{X}$ \\
\hline Total & 6 & 2 & 12 \\
\hline
\end{tabular}

\section{Chrysophyceae}

Chrysococcus rufescens Klebs

Dinobryon divergens Imhof

Pseudokephyrion schilleri (Schiller) Conrad

Total

\begin{tabular}{lll} 
& & $X$ \\
$X$ & $X$ & $X$ \\
$X$ & $X$ & $X$ \\
\hline 2 & 2 & 3 \\
\hline
\end{tabular}

\section{Xantophyceae}

Goniochloris tripus Pascher

Ophiocytium capitatum Wolle

Pseudostaurastrum hastatum (Reinsch.) Chodat

Total

$\mathrm{X}$

\begin{tabular}{ll}
$X$ & $X$ \\
$X$ & $X$ \\
$X$ & 2 \\
\hline 3 &
\end{tabular}

\section{Bacillariophyceae}

Amphora copulata (Kützing) Schoeman \& Archibald

Asterionella formosa Hassal

$\begin{array}{ll} & x \\ X & x \\ x & x \\ & x\end{array}$

Aulacoseira distans (Ehrenberg) Simonsen

Aulacoseira granulata (Ehrenberg) Ralfs (Ehrenberg) Simonsen

Caloneis amphisbaena (Bory) Cleve

Cocconeis placentula Ehrenberg

Cyclotella meneghiniana Kützing

Cyclotella sp.

Cymatopleura solea (Brébisson) W. Smith

Cymbella sp.

$\begin{array}{ll}X & X \\ X & X\end{array}$

X $\quad X \quad x$

Diatoma vulgaris Bory de Saint-Vincent

$\mathrm{X}$

$\mathrm{X}$

$\mathrm{X}$

Fragilaria crotonensis Kitton

Fragilaria ulna var. acus (Kützing) Lange-Bertalot

Fragilaria ulna (Nitzsch) Lange-Bertalot

Fragilaria sp.

Gomphonema truncatum Ehrenberg

Gyrosigma acuminatum (Kützing) Rabenhorst

Gyrosigma cf. fasciola (Ehrenberg) Cleve

Navicula cuspidata (Kützing) Kützing

Navicula sp.

Nitzschia sigmoidea (Nitzsch) W. Smith

Nitzschia sp.

Pinnularia maior (Kützing) Cleve

Pinnularia sp.

Stauroneis anceps Ehrenberg

$\mathrm{X}$

\begin{tabular}{lll}
$X$ & $X$ & $X$ \\
$X$ & $X$ & $X$ \\
$X$ & $X$ & $X$ \\
& & $X$ \\
$X$ & $X$ & $X$ \\
$X$ & & $X$ \\
\hline & & $X$ \\
\hline 11 & 14 & $X$ \\
\hline
\end{tabular}




\begin{tabular}{|c|c|c|c|}
\hline Group, species & Krajskie & Oko & Miejsce \\
\hline \multicolumn{4}{|l|}{ Cryptophyceae } \\
\hline Cryptomonas sp. & $\mathrm{X}$ & & \\
\hline Total & 1 & 0 & 0 \\
\hline \multicolumn{4}{|l|}{ Dinophyceae } \\
\hline Ceratium hirundinella (O.F. Müller) Bergh & & $\mathrm{X}$ & $\mathrm{X}$ \\
\hline Glochidinium penardiforme (Lindermann) & & & $\mathrm{X}$ \\
\hline Kolkwitziella acuta (Apstein) Elbrachter & & $\mathrm{X}$ & \\
\hline Peridinium gatunense Nygaard & $\mathrm{X}$ & & \\
\hline Peridinium sp. & & & $\mathrm{X}$ \\
\hline Peridiniopsis polonicum (Woloszynska) Bourrelly & $\mathrm{X}$ & $\mathrm{X}$ & \\
\hline Tyrannodinium berolinense Calado, Craveiro, Daugbjerg et Moestrup & $\mathrm{X}$ & $\mathrm{X}$ & $\mathrm{X}$ \\
\hline Total & 3 & 4 & 4 \\
\hline
\end{tabular}

\section{Euglenophyta}

Euglena acus Ehrenberg

Euglena clara Skuja

Euglena oxyuris Schmarda

Euglena rostrata Ehrenberg

Euglena spirogyra Ehrenberg

Euglena tripteris (Dujardin) Klebs

Euglena viridis Ehrenberg

Lepocinclis fusiformis (Carter) Lemmermann emend. Conrad

Lepocinclis globulus Perty

Lepocinclis playfairiana Deflandre

Monomorphina pyrum (Ehrenebrg) Mereschkowski

Phacus acuminatus Stokes

Phacus caudatus Hübner

Phacus curvicauda Svirenko

Phacus longicauda (Ehrenberg) Dujardin

Phacus triqueter (Ehrenberg) Dujardin (Pochmann)

Phacus undulatus (Skvortzov) Pochmann

Phacus sp.

Strombomonas acuminata (Schmarda) Deflandre

Trachelomonas allia Drezepolski

Trachelomonas hispida (Perty) Stein

Trachelomonas intermedia P.A.Dangeard

Trachelomonas nigra Svirenko

Trachelomonas oblonga Lemmermann

Trachelomonas planctonica Swirenko

Trachelomonas volvocina Ehrenberg

Total

da) Deflandre

\section{Chlorophyceae}

Actinastrum hantzschii Lagerheim

Botryococcus braunii Kützing

Closteriopsis longissima (Lemmermann) Lemmermann

Coelastrum astroideum De Notaris

Coelastrum microporum Naegeli in A. Braun

Coelastrum reticulatum (P.A.Dangeard) Senn

Coenocystis sp.

Crucigenia fenestrata (Schmidle) Schmidle

Crucigenia tetrapedia (Kirchner) W. \& G.S. West

Crucigeniella apiculata (Lemmerman) Komarek

Desmodesmus armatus (Chodat) Hegewald

Desmodesmus intermedius (Chodat) Hegewald

Desmodesmus opoliensis (P. Richter) Hegewald

\begin{tabular}{|c|c|c|c|}
\hline & $x$ & $X$ & $x$ \\
\hline & & $X$ & $X$ \\
\hline \multirow[t]{6}{*}{ ermann } & $x$ & & $x$ \\
\hline & $X$ & & $X$ \\
\hline & $X$ & $X$ & $x$ \\
\hline & & $X$ & \\
\hline & & $X$ & \\
\hline & & & $x$ \\
\hline \multirow[t]{5}{*}{ 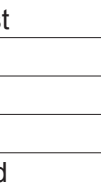 } & $X$ & $X$ & \\
\hline & $X$ & $X$ & $x$ \\
\hline & & & $X$ \\
\hline & & $X$ & $x$ \\
\hline & $X$ & $X$ & $X$ \\
\hline
\end{tabular}




\begin{tabular}{|c|c|c|c|}
\hline Group, species & Krajskie & Oko & Miejsce \\
\hline Desmodesmus spinosus (Chodat) Hegewald & $\mathrm{X}$ & $\mathrm{X}$ & $\mathrm{X}$ \\
\hline Desmodesmus sp. & $\mathrm{X}$ & $\mathrm{X}$ & \\
\hline Dictyosphaerium pulchellum Wood & $X$ & $\mathrm{X}$ & $X$ \\
\hline \multicolumn{4}{|l|}{ Dictyosphaerium tetrachotomum Printz } \\
\hline Kirchneriella dianae (Bohlin) Comas Gonzalez & & $\mathrm{X}$ & $\mathrm{X}$ \\
\hline Lagerheimia genevensis (Chodat) Chodat & & $\mathrm{X}$ & \\
\hline Micractinium pusillum Fresenius & $\mathrm{X}$ & $\mathrm{X}$ & $\mathrm{X}$ \\
\hline Monoraphidium arcuatum (Korshikov) Hindák & & $\mathrm{X}$ & $\mathrm{X}$ \\
\hline Monoraphidium contortum (Thuret) Komàrková- Legnerová & & $\mathrm{X}$ & \\
\hline Oocystis sp. & & $\mathrm{X}$ & $\mathrm{X}$ \\
\hline Pediastrum boryanum (Turpin) Mengehini & $\mathrm{X}$ & $\mathrm{X}$ & $\mathrm{X}$ \\
\hline Pediastrum duplex Meyen & $X$ & $\mathrm{X}$ & $\mathrm{X}$ \\
\hline Pediastrum obtusum Lucks & & & $\mathrm{X}$ \\
\hline Pediastrum simplex Meyen & & & $\mathrm{X}$ \\
\hline Pediastrum tetras (Ehrenberg) Ralfs & $\mathrm{X}$ & $\mathrm{X}$ & $\mathrm{X}$ \\
\hline Polyedriopsis spinulosa (Schmidle) Schmidle & $\mathrm{X}$ & & \\
\hline Scenedesmus acuminatus (Lagerheim) Chodat & $\mathrm{X}$ & $\mathrm{X}$ & $\mathrm{X}$ \\
\hline Scenedesmus ecornis (Ehrenberg) Chodat & $\mathrm{X}$ & $\mathrm{X}$ & $\mathrm{X}$ \\
\hline Scenedesmus denticulatus Lagerheim & $\mathrm{X}$ & $\mathrm{X}$ & $\mathrm{X}$ \\
\hline Scenedesmus naegelii Brébisson & & & $\mathrm{X}$ \\
\hline Scenedesmus sp. & & $\mathrm{X}$ & $\mathrm{X}$ \\
\hline Sphaerocystis sp. & & & $\mathrm{X}$ \\
\hline Tetrastrum glabrum (Roll) Ahlstrim et Tiffany & $\mathrm{X}$ & & $\mathrm{X}$ \\
\hline Tetrastrum komarekii Hindak & & & $\mathrm{X}$ \\
\hline Tetaredron caudatum (Corda) Hansgirg & $\mathrm{X}$ & $\mathrm{X}$ & \\
\hline Total & 20 & 26 & 29 \\
\hline \multicolumn{4}{|l|}{ Conjugatophyceae } \\
\hline Closterium sp. & $\mathrm{X}$ & $\mathrm{X}$ & $\mathrm{X}$ \\
\hline Cosmarium sp. & & & $\mathrm{X}$ \\
\hline Staurastrum sp. & & $\mathrm{X}$ & $\mathrm{X}$ \\
\hline Total & 1 & 2 & 3 \\
\hline Total of all species & 65 & 66 & 88 \\
\hline
\end{tabular}

Pinnularia subgibba Krammer, Surirella brebissonii Krammer \& Lange-Bertalot. Also present in this reservoir was Peridiniopsis kevei Grigorszky \& Vasas (Dinophyceae), which is classified as an invasive species (Owsianny and Grabowska 2009). The presence of planktonic Cyanophyceae (Microcystis aeruginosa Kütz and Woronichinia naegeliana Unger (Elenkin)) and Chrysophyceae (Synura uvella Ehr. emend. Korsikov) in Miejsce oxbow lake allowed this reservoir to be classified as eutrophic water with a high content of organic matter.

In general, among the investigated phytoplankton species, cosmopolitan ones were also found, some of them considered to be resistant to contamination by heavy metals. Among the species found in Krajskie were, e.g. filamentous Cyanophyceae Oscillatoria sp.; Bacillariophyceae Nitzschia sigmoidea (Nitzsche) W. Smith; Chlorophyceae Scenedesmus species: S. acu- minatus (Lagerheim) Chodat, S. denticulatus Lagerheim, and S. ecornis (Ehrenberg) Chodat. It is worth mentioning, within the Euglenophyta, the presence of Euglena mutabilis Schmitz (Starmach), which was also found in Krajskie. It is a species resistant to high concentrations of cadmium, nickel, aluminum and iron (Pawlik-Skowrońska 2002a). In addition, many studies showed that Chlorophyceae tolerate high concentrations of heavy metals such as $\mathrm{Cu}$ and $\mathrm{Zn}$ (Shubert et al. 2001; Takamura et al. 1989; Vymazal 1995).

Algae can be present in habitats contaminated with heavy metals, because of their high resistance (creation of biochemical and physiological mechanisms), and also because of the effect of the low bioavailability of the metals despite their high concentration (PawlikSkowrońska 2002a). Bioavailability of toxic metals to algae is difficult to ascertain in field studies due to the complexity of aquatic habitats. The indicator of heavy 
metal bioavailability to algae is determined by knowing the level induced by certain metals and metalloids, thiol peptides - phytochelatins, whose synthesis is activated by metal penetration into the cells of algae (Pawlik-Skowrońska 2002a). Species living in ecosystems contaminated by heavy metals must adapt to these unfavourable conditions. Adaption evolves because the heavy metals possess specific biochemical and physiological characters of the organism. Therefore, the resistance of algae is defined as the tolerance to metals - survival, development and reproduction, despite a considerable amount of metals in the cells - or as the ability to remove a harmful element. It is also proved that the existence of high concentrations of $\mathrm{Ca}$ and $\mathrm{Mg}$ cations can reduce the penetration of large amounts of $\mathrm{Pb}$ and $\mathrm{Zn}$ in the cells (Pawlik-Skowrońska 2002b). However, plankton can suffer a toxic effect despite its tolerance mechanisms (Vega-Lopez et al. 2013). Some studies revealed also that relatively high concentrations of heavy metals in reservoirs, mainly $\mathrm{Cu}, \mathrm{Zn}$ and $\mathrm{Mn}$, do not inhibit the growth of algae, but others showed that it caused the development of some species more resistant to contamination (Pasternak 1971; Ligęza and Wilk-Woźniak 2011). High concentrations of Fe may be associated with a relatively high abundance of Cyanophyceae or Bacillariophyceae species such as Asterionella formosa and Aulacoseira granulata or Euglenophyta species such as Trachelomonas volvocina (Vymazal 1995). The presence of these species was recorded in all three reservoirs.

The index of taxa similarity was defined for phyto- and zooplankton (Table 4). The index showed the highest resemblance for Oko and Miejsce reser-

Table 4. The taxonomic similarity factor for three oxbow lakes calculated based on the composition of the groups: Cyanophyceae, Bacillariophyceae, Euglenophyta, Chlorophyceae, Rotifera, Cladocera and Copeoda. Description: Pko - taxonomic resemblance between Krajskie and Oko reservoirs, Pmk - taxonomic resemblance between Oko and Miejsce, Pmk - taxonomic resemblance between Miejsce and Krajskie

\begin{tabular}{lccc}
\hline \multirow{2}{*}{ Group } & \multicolumn{3}{c}{2011} \\
\cline { 2 - 4 } & Pko [\%] & Pom [\%] & Pmk [\%] \\
\hline Cyanophyceae & 25 & 28.6 & 44.4 \\
\hline Bacillariophyceae & 48 & 72 & 54.5 \\
\hline Euglenophyta & 48.5 & 61.5 & 54.5 \\
\hline Chlorophyceae & 69.6 & 69.1 & 65.3 \\
\hline Rotifera & 69.8 & 65.1 & 65 \\
\hline Cladocera & 52.6 & 74.1 & 60 \\
\hline Copepoda & 0 & 0 & 22.2 \\
\hline
\end{tabular}

voirs among Bacillariophyceae and Euglenophyta and the lowest between Oko and Krajskie. Among Cyanophyceae the index of taxa similarity showed the highest resemblance for Miejsce and Krajskie. However, the same index showed percentages of similarity for Chlorophyceae taxa for all reservoirs. It seems that the structure of Chlorophyta species was less specific, whereas the structures of Bacillariophyceae and Euglenophyta species were more specific for oxbow lakes and for each reservoir.

In the case of zooplankton communities in 2011, the presence of 29 species in Krajskie, 38 in Oko and 40 in Miejsce was revealed. Many cosmopolitan species were found, e.g. Polyarthra vulgaris Carlin and Keratella cochlearis Goss, and among the Cladocerans were noted species characteristic for slightly saline water, e.g. Simocephalus (Echinocaudus) exspinosus De Geer, Alona rectangula Sars and Leydigia leydigi Schoedler.

Usually, the toxic effects of heavy metals on plankton organisms can be seen as deformations of spines for K. cochlearis or deformations of blades for P. vulgaris (Wilk-Woźniak et al. 2011). However, in the case of zooplankton research of complex oxbow lakes, so-called "Wiślisko", no teratogenic forms of plankton organisms were observed. In each reservoir the Rotifers, the group most resistant to contamination (Jak et al. 1996), dominated. There was a smaller share of the number of Cladocera and Copepoda species, groups particularly vulnerable to contamination with heavy metals (Sarma et al. 2007).

The index of taxa similarity was also calculated for zooplankton (Table 4). In the case of Rotifera, similar to Chlorophyceae, this parameter did not differ among the oxbow lakes, but they varied for Cladocera and Copepoda. The highest resemblance was found for the Cladocera species structure in Oko and Miejsce and the lowest for Oko and Krajskie reservoirs. Among Copepoda the index of taxa similarity showed resemblance for Miejsce and Krajskie. Cladocera and Copepoda are the groups of zooplankton less resistant to contamination compared to Rotatoria (Sarma et al.2007), which explains for both planktonic groups why the index of taxa similarity varied among oxbow lakes, but did not vary for Rotatoria.

The index of taxa similarity indicated that the studied reservoirs are varied in species structure of phyto- and zooplankton but not for all groups. The variation of species indicates the different type of habitat of all three oxbow lakes, even when they are close 
to each other, which was also stated by other authors (Cottenie et al. 2001). The differences in the structure of plankton were rather the reflection of chemical features of the waters (e.g. conductivity, chlorides, ammonia, nitrates) than sediment contamination. However, to be sure that the exclusion of the impact of heavy metals migrating from the bottom sediments to the plankton organisms (especially during the summer) is correct (e. g. by investigating other possibilities such as dissolved organic carbon, sulphide, etc. able to modify the bioavailability of heavy metals), further research is required.

\section{Conclusions}

- Heterogeneous contamination with heavy metals $(\mathrm{Zn}, \mathrm{Cd}, \mathrm{Cu}, \mathrm{Pb}, \mathrm{Cr}$ and $\mathrm{Fe}$ ) of bottom sediments in three adjacent oxbow lakes was the result of the impact of contaminated waters of the River Vistula.

- Effects of heavy metal contamination can be mitigated by relatively good overall parameters of water with $\mathrm{pH}$ above neutral.

- Deficiency of oxygen close to the bottom occurring during the summer might create good conditions for the remobilization of heavy metals from the sediments.

- Significant statistical differences between oxbow lakes were noted for $\mathrm{pH}$, conductivity and chlorides. These differences were explained by the impact of the River Vistula.

- The index of taxa similarity showed higher resemblance of species for Bacillariophyceae, Euglenophyta, and Cladocera in Oko and Miejsce oxbow lakes (lack of direct connection with the River Vistula) and a lower percentage of similarity with Krajskie oxbow lake (direct connection with the River Vistula). These observations were explained by significant differences mostly in chlorides and conductivity.

- The index of taxa similarity was similar for all three oxbow lakes for Chlorophyta and Rotatoria.

- It is necessary to conduct a comprehensive study of the migration of metals to the water in order to determine the real ecotoxicological risk for the microflora and microfauna of oxbow lakes „Wiślisko” during the summer stagnation.

\section{Acknowledgments}

I am thankful to Elżbieta Wilk-Woźniak and Agnieszka Pociecha (Institute of Nature Conservation Polish Academy of Sciences) for kindly providing the data of phyto- and zooplankton analysed in 2010 and 2011 in the three reservoirs of the River Vistula's oxbow lake near Zator (Oświęcim Basin). The author highly appreciates the valuable comments and suggestions of four anonymous reviewers, which significantly contributed to improving the manuscript.

\section{References}

Atici T., Ahiska S., Altindağ A., Aydin D., 2008, Ecological effects of some heavy metals $(\mathrm{Cd}, \mathrm{Pb}, \mathrm{Hg}, \mathrm{Cr}$ ) pollution of phytoplanktonic algae and zooplanktonic organisms in Sarıyar Dam Reservoir in Turkey, Afr. J. Biotechnol. 7(12): 1972-1977.

Atici T., Obali O., Altindag A., Ahiska S., Aydin D., 2010, The accumulation of heavy metals $(\mathrm{Cd}, \mathrm{Pb}, \mathrm{Hg}, \mathrm{Cr})$ and their state in phytoplanktonic algae and zooplanktonic organisms in Beysehir Lake and Mogan Lake, Turkey, Afr. J. Biotechnol. 9(4): 475-487.

Bajkowska M., 2012, Ocena potencjalnego ekotoksykologicznego oddziaływania metali ciężkich występujących w osadach dennych starorzecza Wisły w rejonie Zatora (Assessment of the potential ecotoxicological effects of bottom sediments contamination with heavy metals in the Vistula's oxbow lake at Zator) [Master's thesis], AGH Kraków, Wydz. GGiOŚ, Kraków, p. 43 (in Polish).

Ciszewski D., Czajka J., 2009, Akumulacja osadów na równinach zalewowych rzek silnie zmienionych antropogenicznie: górna Wisła i Odra (Sediment accumulation on alluvial plains of the heavily impacted river reaches: upper Vistula and Odra, southern Poland), Prz. Geol. 57(7): 576-583 (in Polish, English summary).

Ciszewski D., Aleksander-Kwaterczak U., Kubsik U. Kwadrans J., Pociecha A., Szarek-Gwiazda E., Tłoczek I., Waloszek A., Wilk-Woźniak E., 2011, Interdisciplinary investigations of contamination effects of pond and stream waters and sediments in the Matylda catchment - an attempt to classification, [in:] Zieliński A. (ed.), Interdisciplinary researches in natural sciences, Wyd. IG UJK, Kielce: $29-46$.

Ciszewski D., Pociecha A., Szczęsny B., Wilk-Woźniak E., Zając T., 2012, Wpływ wód Wisły na zanieczyszczenie chronionych starorzeczy w rejonie Spytkowic (Influence of the Vistula River waters on pollution of the protected oxbow lakes at Spytkowice (Southern Poland)), Gór. Geol. 7(2): 115-128 (in Polish, English summary).

Cottenie K., Nuytten N., Michels E., De Meester L. 2001, Zooplankton community structure and environmental conditions in a set of interconnected ponds, Hydrobiologia 442: 339-350. 
Gadzinowska J., 2012, Wpływ rzeki Wisły na zanieczyszczenie osadów dennych metalami ciężkimi jej starorzecza w rejonie Zatora (The impact of the Vistula river on contamination of the bottom sediments with heavy metals in the Vistula's oxbow lake near Zator) [Master's thesis], AGH Kraków, Wydz. GGiOŚ, Kraków, p. 60 (in Polish).

[GIOŚ] Główny Inspektorat Ochrony Środowiska, 2011, Monitoring osadów rzecznych i jeziornych (Monitoring of river and lake sediments), Retrieved from http://ekoinfonet.gios.gov.pl/osady (in Polish).

Greig-Smith H., 1983, Quantative plant ecology, BlackwellOxford Univ. Press, New York, p. 359.

Helios-Rybicka E., 1986, Rola minerałów ilastych w wiązaniu metali ciężkich przez osady rzeczne górnej Wisły (Role of clay minerals in the fixation of heavy metals in bottom sediments of the Upper Vistula River system), Geologia 32: 1-123 (in Polish).

Helios-Rybicka E., 1991, Akumulacja i mobilizacja metali ciężkich w osadach środowiska wodnego: osady datowane jako wskaźnik chronologiczny (Accumulation and mobilization of heavy metals in bottom sediments of the aqueous environment: dated sediments as a chronological indicator), [in:] Proc. of the Conference "Geologiczne aspekty ochrony środowiska”, Wyd. AGH, Kraków: 17-24 (in Polish).

Hermanowicz W., Dojlido J., Dożańska W., Koziorowski B., Zerbe J., 1999, Fizyczno-chemiczne badanie wody i ścieków (Physicochemical analyses of water and sewage), Arkady, Warszawa, p. 556 (in Polish).

Jak R.G., Maas J.I., Scholten M.C.T., 1996, Evaluation of laboratory derived toxic effect concentrations of a mixture of metals by testing freshwater plankton communities in enclosures, Water Res. 30(5): 1215-1227.

Ligęza S., Wilk-Woźniak E., 2011, The occurrence of Euglena pascheri and Lepocinclic ovum bloom in an oxbow Lake in southern Poland under extreme environmental conditions, Ecol. Indicat. 11(3): 925-929.

Lis J., Pasieczna A., 1995, Atlas Geochemiczny Górnego Śląska 1:200 000 (Geochemical Atlas of Upper Silesia 1:200 000), PIG, Warszawa (in Polish).

Łojan E., 2008, Wpływ składników mineralnych na geochemię metali ciężkich w osadach dennych rzeki Wilgi (Influence of mineral components on geochemistry of heavy metals in sediments of the Wilga river) [Dissertation], AGH Kraków, Wydz. GGiOŚ, Kraków, p. 180 (in Polish).

MacDonald D.D, Ingersoll C.G., Berger T.A., 2000, Development and evaluation of consensus-based Sediment Quality Guidelines for freshwater ecosystems, Arch. Environ. Contam. Toxicol. 39(1): 20-31.

Owsianny P.M., Grabowska M., 2009, Bruzdnice Wigier i zbiorników przyległych - gatunki nowe, rzadkie, inwazyjne (Dinoflagelates of Wigry area and adjacent reservoirs - new, rare and invasive species), Retrieved from http://www.wigry.win.pl/konferencja_wodna/Owsianny.pdf. (in Polish).
Pasternak K., 1971, The content of copper, zinc and manganese in the water of the dam reservoir at Goczałkowice and of several other reservoirs, Acta Hydrobiol. 13: 159-177.

Pawlik-Skowrońska B., 2002a, Tajemnice odporności glonów i sinic na toksyczne metale ciężkie (Mystery of algal resistance to heavy metals), Kosmos 51(2): 175-184 (in Polish, English summary).

Pawlik-Skowrońska B., 2002b, Correlations between toxic $\mathrm{Pb}$ effects and production of $\mathrm{Pb}$-induced thiol peptides in the microalga Stichococcus bacillaris, Environ. Pollut. 119(1): 119-127.

Pociecha A., Higgins T., McCarthy T. K., 2010, A preliminary study on the plankton assemblages of Lough Derg (Ireland) during a winter-spring season, Ocean. Hydrob. Stud. 39(3): 145-154.

Sarma S. S. S., Peredo-Alvarez V. M., Nandini S., 2007, Comparative study of the sensitivities of neonates and adults of selected cladoceran (Cladocera: Crustacea) species to acute toxicity stress, J. Environ. Sci. Heal. A 42(10): 1449-1452.

Shobert B., 1974, The influence of water stress on the metabolism of diatoms. I. Osmotic resistance and proline accumulation in Cyclotella meneghiniana, Z. Pflanzenphysiol. 74: 106-120.

Shubert E., Rusu A-M., Bartok K., Moncrieff C.B., 2001, Distribution and abundance of adaphic algae adapted to highly acidic metal rich solids, Nova Hedwigia 123: 411425.

Siemińska J., Bąk M., Dziedzic J., Gąbka M., Gregorowicz P., Mrozińska T., Pełechaty M., Owsianny P.M., Pliński M, Witkowski A., 2006, Red list of the algae in Poland, [in:] Mirek Z., Zarzycki K., Wojewoda W., Szelaj Z. (eds), Red list of plants and fungi in Poland, W. Szafer Institute of Botany PAS, Kraków: 37-52.

Skowroński T., Kalinowska R., Pawlik-Skowrońska B., 2002, Glony środowisk zanieczyszczonych metalami ciężkimi (Alga in heavy metal-polluted environments), Kosmos 51(2): 165-173 (in Polish, English summary).

Skowroński T., Rzeczycka M., 1980, Effect of high zinc concentrations on the growth of Stichococcus bacillaris and Chlorella vulgaris, Acta Microb. Pol. 29: 389-396.

Smith S.L., MacDonald D.D., Keenleyside K.A., Ingersoll C.G., Field L.J., 1996, A preliminary evaluation of sediment quality assessment values for freshwater ecosystems, J. Great Lakes Res. 22(3): 624-638.

Takamura N., Kasai F., Watanabe M. M., 1989, Effects of Cu, $\mathrm{Cd}$ and $\mathrm{Zn}$ on photosynthesis of freshwater benthic algae, J. Appl. Phycol. 1(1): 39-52.

Vega-López A., Ayala-López G., Posadas-Espadas B. P., Olivares-Rubio H. F., Dzul-Caamal R., 2013, Relations of oxidative stress in freshwater phytoplankton with heavy metals and polycyclic aromatic hydrocarbons, Comp. Biochem. Physiol. Part A 165(4): 498-507.

Vymazal J., 1995, Algae and element cycling in wetlands, CRC Press/Lewis Publ., Boca Raton: p. 689. 
Watson S. B., McCauley E., Downing J.A., 1997, Patterns in phytoplankton taxonomic composition across temperate lakes of differing nutrient status, Limnol. Oceanogr. 42(3): 487-495.

Wilk-Woźniak E., 2009, Zmiany populacyjne w zbiorowiskach glonów planktonowych oraz ich strategie życiowe w warunkach ekosystemów wodnych sztucznie zmienionych (Changes in phytoplankton communities and the life strategies of planktonic algae in artificially changed aquatic ecosystems), Studia Naturae 55: 1-132 (in Polish).

Wilk-Woźniak E., Pociecha A., Ciszewski D., AleksanderKwaterczak U., Walusiak E., 2011, Phyto- and zooplankton in fishponds contaminated with heavy metal runoff from a lead-zinc mine, Oceanol. Hydrobiol. Studies 40(4): 77-85.
Wilk-Woźniak E., Ligęza S., Shubert E., 2013, Effect of water quality on phytoplankton structure in oxbow lakes under anthropogenic and non-anthropogenic impacts, CLEAN - Soil Air Water, Retrieved from DOI. 10.1002/ clen.201200214.

Zając T., Pociecha A., Wilk-Woźniak E., Zając K., Bielański W., Ciszewski D., Florek J., Gołąb M., Guzik M., Lipińska A., Myszka R., Najberek K., Potoczek M., Walusiak E., Szczęsny B., 2013, Analiza właściwego stanu ochrony starorzecza na przykładzie kompleksu starorzeczy „Wiśliska" - obszar Natura 2000 PLH 120084 (Analysis of the conservation status of the "Wiśliska" oxbow lakes Natura 2000 site PLH 120084), Chrońmy Przyr. Ojcz. 68 (2): 116-133 (in Polish). 